\title{
Accelerated telomere attrition in children and teenagers with $\alpha_{1}$-antitrypsin deficiency
}

\author{
Amparo Escribano ${ }^{1,2,3,4,10}$, Sara Pastor ${ }^{3,5,10}$, Ana Reula ${ }^{3,5}$, Silvia Castillo ${ }^{1,2,3}$, \\ Silvia Vicente ${ }^{3}$, Francisco Sanz ${ }^{6}$, Francisco Casas ${ }^{4,7}$, María Torres ${ }^{4,8}$, \\ Estrella Fernández-Fabrellas ${ }^{6}$, Pilar Codoñer-Franch ${ }^{1,9}$ and Francisco Dasi $i^{3,4,5}$
}

Affiliations: 'Dept of Paediatrics, Obstetrics and Gynecology, School of Medicine, University of Valencia, Valencia, Spain. ${ }^{2}$ Paediatrics Pneumology Unit, Hospital Clínico Universitario Valencia, Valencia, Spain. ${ }^{3}$ Fundación Investigación Hospital Clínico Universitario de Valencia/Instituto de Investigación Sanitaria INCLIVA, Valencia, Spain. ${ }^{4}$ Spanish Registry for Alpha-1 antitrypsin Deficiency (REDAAT), Barcelona, Spain. ${ }^{5}$ Dept of Physiology, School of Medicine, University of Valencia, Valencia, Spain. ${ }^{6}$ Pulmonology Unit, Consorcio Hospital General Universitario de Valencia, Valencia, Spain. ${ }^{7}$ Pulmonology Unit, Hospital Universitario San Cecilio de Granada, Granada, Spain. ${ }^{8}$ Pulmonology Unit, Complexo Universitario de Vigo, Pontevedra, Spain. ${ }^{9}$ Paediatrics Unit, Hospital Universitario Dr. Peset Valencia, Valencia, Spain. ${ }^{10}$ These authors contributed equally.

Correspondence: Francisco Dasí, Fundación Investigación Hospital Clínico Universitario de Valencia/Instituto de Investigación Sanitaria INCLIVA, c/ Menéndez y Pelayo 4, 46010 Valencia, Spain. E-mail: Francisco.Dasiduv.es

ABSTRACT Numerous studies have shown that oxidative stress accelerates telomere shortening in several lung pathologies. Since oxidative stress is involved in the pathophysiology of $\alpha_{1}$-antitrypsin deficiency (AATD), we hypothesised that telomere shortening would be accelerated in AATD patients. This study aimed to assess telomere length in AATD patients and to study its association with $\alpha_{1}$-antitrypsin phenotypes.

Telomere length, telomerase activity, telomerase reverse transcriptase ( $h T E R T)$ expression and biomarkers of oxidative stress were measured in 62 children and teenagers (aged 2-18 years) diagnosed with AATD and 18 controls (aged 3-16 years).

Our results show that intermediate-risk (MZ; SZ) and high-risk (ZZ) AATD patients have significantly shorter telomeres and increased oxidative stress than controls. Correlation studies indicate that telomere length was related to oxidative stress markers in AATD patients. Multiple hypothesis testing revealed an association between telomere length, telomerase activity, $h T E R T$ expression and AATD phenotypes; highrisk patients showed shorter telomeres, lower hTERT expression and decreased telomerase activity than intermediate-risk and low-risk patients.

AATD patients show evidence of increased oxidative stress leading to telomere attrition. An association between telomere and $\alpha_{1}$-antitrypsin phenotypes is observed suggesting that telomere length could be a promising biomarker for AATD disease progression.

@ERSpublications

Patients with $\alpha_{1}$-antitrypsin deficiency have shorter telomeres than healthy individuals http://ow.ly/8NHS300zz5A

This article has supplementary material available from erj.ersjournals.com

Received: Jan 232016 | Accepted after revision: May 172016 | First published online: July 072016

Support statement: This work was supported by 2012 INCLIVA intramural, ISCIII PI14/02162 grants and European Regional Development Funds. Funding information for this article has been deposited with Open Funder Registry.

Conflict of interest: None declared.

Copyright OERS 2016 


\section{Introduction}

$\alpha_{1}$-antitrypsin deficiency (AATD) is a rare, genetic and hereditary condition that leads to low plasma levels of the $\alpha_{1}$-antitrypsin antitrypsin (AAT) protein, significantly increasing the risk of chronic obstructive pulmonary disease (COPD) and liver disease in these patients [1].

The AAT protein is encoded by the SERPINA1 gene, and more than 100 variants have been described in this locus, most of them without a defined biological meaning. The most common pathogenic variants are the $\mathrm{S}$ and $\mathrm{Z}$ forms (as opposed to the normal $\mathrm{M}$ allele) [2].

A great variability in the severity of symptoms found in AATD patients has been observed, and neither plasma levels of AAT nor phenotype are sufficient to identify those patients who will develop severe liver or lung disease indicating that there must be other mechanisms besides the protease-antiprotease imbalance that contribute to the development of emphysema or liver disease [3]. Therefore, the identification of other risk factors involved in the progression of lung or liver disease may help us understand the variability of the symptoms observed in these patients and the ability to counter them.

Oxidative stress has been shown to be a contributing factor to the development of liver and lung damage in AATD animal models [4-6]. Our research group recently demonstrated in asymptomatic (normal lung function and liver damage parameters) AATD children, that oxidative stress produced by a reduction in antioxidant defences is involved in the pathophysiology of AATD and is associated with the risk of developing lung and/or liver disease [7]. Overall, these data might suggest that oxidative stress could play a role in the decline in lung and/or liver function in AATD patients.

Epidemiological studies have revealed that loss of telomere integrity is an important factor in the decay of physiological functions associated with ageing, inflammation and several chronic illnesses [8, 9]. In this respect, it has been suggested that telomere length may be used as a biomarker of chronic oxidative stress and inflammation $[10,11]$.

Telomeres are DNA protein structures located at the end of chromosomes that protect them from degradation and end-to-end fusion. Telomeric sequences are synthesised by a specialised reverse transcriptase called telomerase, an enzyme composed of several subunits including the telomerase RNA $(h T R)$ and the catalytic subunit telomerase reverse transcriptase ( $h T E R T)$. By using $h T R$ as a template, hTERT adds telomeric repeats to the $3^{\prime}$-strands of chromosomes to form the telomeres. It has been shown that the rate of telomere shortening is influenced by both genetic, as well as environmental factors [11, 12]. Telomere state depends not only on its length but also on the composition of the telomere cap and telomerase activity. Any change to any of these factors could jeopardise telomeric integrity, which would lead to loss of telomere function [13].

Numerous studies have shown that oxidative stress accelerates telomere shortening in several lung pathologies [14], and it has been found to be associated with emphysema risk in COPD patients [15-17]. Therefore, it seems relevant to investigate telomere shortening in AATD, a condition characterised by chronic oxidative stress and inflammation.

The aim of this study was to determine whether AATD was associated with reduced telomere length and to explore its association with AAT phenotypes. In addition, telomerase activity and $h T E R T$ expression were determined to increase our knowledge on telomere biology. We hypothesised that AATD patients would have shorter telomeres, reduced telomerase activity and reduced $h T E R T$ expression compared to controls. Moreover, in AATD patients, telomere length would be related to AAT phenotypes.

\section{Materials and methods}

Study subjects

62 patients and 18 controls were recruited by genetic counselling from the paediatrics units of the Hospital Clínico Universitario Valencia and Hospital Universitari Doctor Peset (both Valencia, Spain) from May 2011 to January 2016. Patients had a familial history of AATD. Inclusion criteria were: 1) patients diagnosed with AATD according to the American Thoracic Society/European Respiratory Society recommendations [18]; and 2) control individuals with MM phenotype and no history or clinical findings that suggested a pulmonary or hepatic pathology. Exclusion criteria were the following: 1) COPD or hepatic disease not related to AATD; 2) patients with diseases related to oxidative stress, such as rheumatoid arthritis, lupus erythematosus, inflammatory bowel disease, diabetes mellitus, cardiac dysfunction and neoplasms; 3) active fever or infection; 4) surgery $<3$ months prior to the sample collection; 5) intense physical exercise $<2$ days prior to sample collection; 6) treatment with antioxidants 3 months prior to sample collection; and 7) acetaminophen (paracetamol) consumption for at least 1 month before blood sampling.

All samples were analysed and blinded with respect to the patients' clinicopathological status. The study was approved by the research ethics committee of the Hospital Clínico Universitario Valencia. Procedures 
were explained to the children, young teenagers and parents. Written informed consent was obtained from all participants. In the case of the children, the informed consent form was signed by their parents.

\section{Anamnesis and physical examination}

Anthropometric measurements were performed using standard techniques. Body mass index (BMI) was calculated as $\mathrm{kg} \cdot \mathrm{m}^{-2}$. Pulmonary function testing was performed by spirometry, computed tomography (CT) and diffusing capacity of the lung for carbon monoxide. Liver function was determined by measuring aspartate aminotransferase (AST), alanine aminotransferase (ALT) and $\gamma$-glutamyl transferase (GGT). Serum concentration of AAT was measured by nephelometry. Pulmonary, liver and AAT normality values are indicated in table $1[19,20]$. AAT phenotypes were determined by isoelectric focusing of serum samples as previously described [21].

\section{Blood sampling and DNA/RNA isolation}

Peripheral venous blood was collected from patients and healthy volunteers after $12 \mathrm{~h}$ fasting in $4 \mathrm{~mL} \mathrm{BD}$ Vacutainer CPT with sodium heparin (Becton Dickinson, Franklin Lakes, NJ, USA) and peripheral blood mononuclear cells (PBMC) separated according to the manufacturer's instructions.

Parallel isolation of DNA and RNA from PBMC was performed using the NucleoSpin Triprep (Macherey Nagel, Düren, Germany) according to the manufacturer's recommendations.

For serum separation, whole blood was collected in serum separation tubes (Becton Dickinson), allowed to clot at room temperature for $30 \mathrm{~min}$ and centrifuged at $2000 \times \mathrm{g}$ for $10 \mathrm{~min}$ at $4^{\circ} \mathrm{C}$. The resulting supernatant was collected and stored at $-80^{\circ} \mathrm{C}$ until analysis.

\section{Oxidative stress parameters}

Oxidative stress parameters were measured as previously described [7]. For further details refer to the supplementary material.

\section{Telomere length assay}

Measurement of telomere length was performed by quantitative PCR as previously described [22]. For further details refer to the supplementary material.

\section{Telomerase activity}

Telomerase activity was measured using the TRAPeze XL Telomerase Detection Kit (Millipore, Billerica, MA, USA) according to the manufacturer's instructions. For further details refer to the supplementary material.

\section{hTERT gene expression}

Quantitative detection of hTERT mRNA was performed by reverse transcription quantitative PCR. For further details refer to the supplementary material.

TABLE 1 Demographics and clinical characteristics in $\alpha_{1}$-antitrypsin deficiency patients and controls

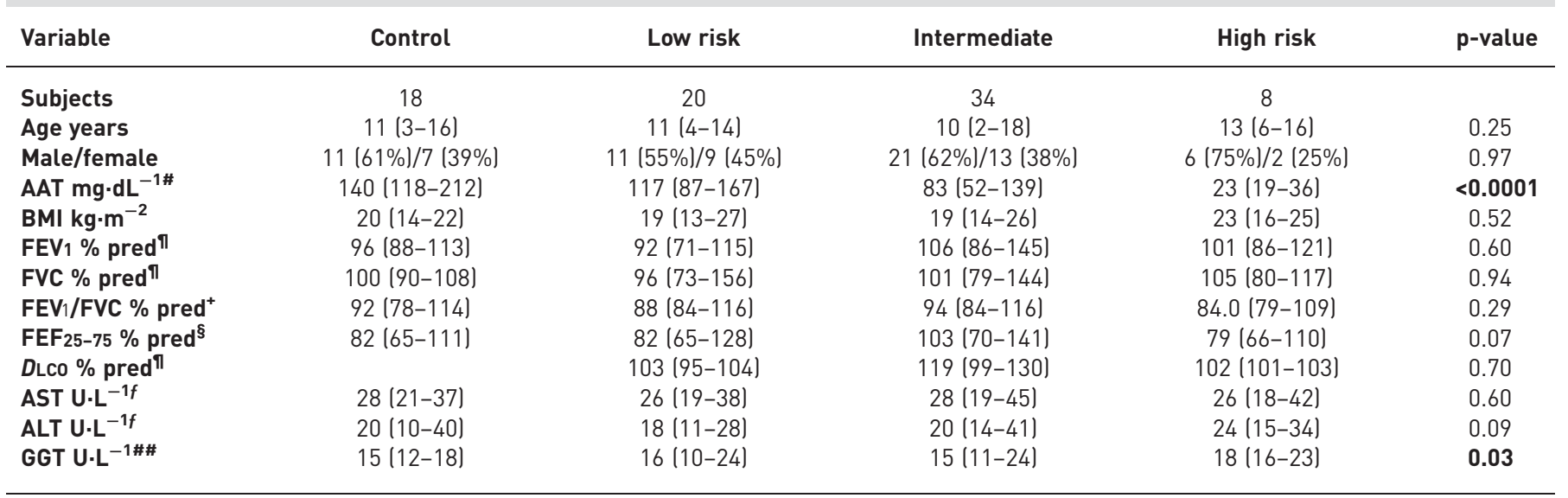

Data are presented as median (range), unless otherwise stated. Bold indicates statistical significance $(p<0.05)$ after application of the KruskalWallis test. Comparison of proportions was performed using the Chi-squared test. AAT: $\alpha_{1}$-antitrypsin; BMI: body mass index; FEV1: forced expiratory volume in $1 \mathrm{~s}$; FVC: forced vital capacity; FEF25-75: forced expiratory flow at 25-75\% of FVC; DLCo: diffusing capacity of the lung for carbon monoxide; AST: aspartate aminotransferase; ALT: alanine aminotransferase; GGT: $\gamma$-glutamyl-transferase. ${ }^{\#}$ : normal range $90-200 \mathrm{mg} \cdot \mathrm{dL}^{-1}$;

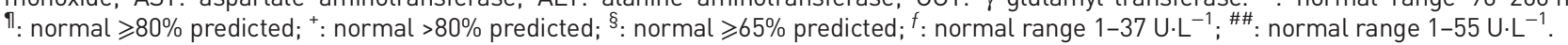




\section{Statistical analyses}

Demographic and clinical data are expressed as median and range (minimum and maximum values). Assessment of normality was performed by the Shapiro-Wilk normality test. Biomarkers of oxidative stress are expressed as median and interquartile range (IQR). Median comparison between groups of continuous variables was performed using the Kruskal-Wallis non-parametric test. Telomere length, telomerase activity and hTERT mRNA expression data followed a normal distribution and differences were assessed using ANOVA. Multiple hypothesis testing was performed (Holm-Sidak and Dunn's multiple comparisons tests) to identify the significant pairwise differences among groups. The Chi-square test was applied for proportion comparisons. Correlations between telomere length and oxidative stress markers were assessed using Spearman's rank correlation test. A two-tailed p-value $<0.05$ was considered statistically significant. Statistical analyses were performed using the GraphPad Prism 6.00 Software (GraphPad, La Jolla, CA, USA).

\section{Results}

\section{Demographic and clinical data}

Demographic and clinical characteristics of patients and controls included in the study are presented in table 1.

Patients were categorised according to their AAT phenotype in three different risk groups for developing lung and/or liver damage: low risk (MS; SS), intermediate risk (MZ; SZ) and high risk (ZZ) [7]. A control group of healthy volunteers with the MM phenotype was also included in the study. 18 (22.5\%) of the 80 subjects included in the study had the MM phenotype, 20 (25.0\%) had low-risk, 34 (42.5\%) had intermediate-risk and eight (10\%) had high-risk phenotypes.

All the subjects included in the study were clinically healthy according to both their physical status and their pulmonary and liver tests (table 1). CT scans revealed no signs of lung damage in any of the individuals included in the study (data not shown). Regarding liver damage markers, there were no differences in AST and ALT levels between groups. A significant increase $(p=0.03)$ in serum GGT in high-risk patients was observed, although none of the patients showed GGT values above normal range. There were no differences in age, sex, BMI and lung function tests across the groups. As expected, significant differences were observed in the AAT levels between groups $(\mathrm{p}<0.0001)$.

\section{Telomere shortening in circulating PBMC in AATD patients}

Significant differences in telomere length were observed between groups $(p=0.0006)$ (figure 1). Multiple analysis testing revealed that intermediate-risk and high-risk patients had significantly lower telomere length ( $\mathrm{p}=0.042$ and $\mathrm{p}=0.026$, respectively) than the control group, whereas no significant differences were observed between the low-risk patients and the control group $(\mathrm{p}=0.389)$. When AATD patients were compared to each other, telomeres were significantly shorter in intermediate-risk and high-risk groups than in the low-risk group ( $\mathrm{p}=0.024$ and $\mathrm{p}<0.0001$, respectively). No significant differences in telomere length were observed between the high-risk and intermediate-risk groups $(\mathrm{p}=0.102)$.

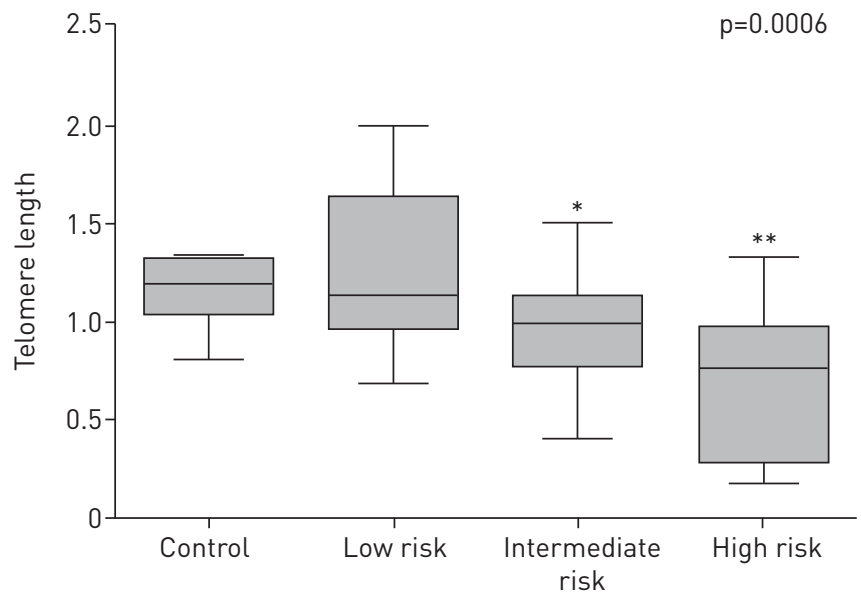

FIGURE 1 Telomere shortening in circulating peripheral blood mononuclear cells in $\alpha_{1}$-antitrypsin deficiency (AATD) patients. Boxplots show telomere length and AATD risk groups. Data are presented as median and interquartile range. Significant differences in telomere length were observed between groups $(p=0.0006)$. Telomere length was significantly decreased in intermediate-risk ( $p=0.042)$ and high-risk patients ( $p=0.026)$ compared to controls. No significant differences were observed between the low-risk patients and the controls $(p=0.389) .{ }^{*}: p \leqslant 0.05 ; * *: p \leqslant 0.01$. 
Decreased hTERT expression in PBMC of AATD patients

Significant differences in $h T E R T$ expression were observed between groups $(\mathrm{p}=0.0002)$ (figure 2). Multiple analysis testing showed that when compared with that for the control group, intermediate-risk and high-risk patients had significantly lower $h T E R T$ expression ( $\mathrm{p}=0.001$ and $\mathrm{p}=0.0005$, respectively), whereas no significant differences were observed in the comparison with the low-risk group $(\mathrm{p}=0.225)$. When AATD patients were compared to each other, $h T E R T$ expression was significantly lower in the high-risk than in the low-risk group $(\mathrm{p}=0.03)$. No significant differences in $h T E R T$ expression were observed between the high-risk and the intermediate-risk groups $(\mathrm{p}=0.302)$ or between the intermediate-risk and the low-risk groups $(\mathrm{p}=0.129)$.

\section{Decreased telomerase activity in PBMC of AATD patients}

Significant differences in telomerase activity were observed between groups $(\mathrm{p}<0.0001)$ (figure 3$)$. When compared to the control group, telomerase activity was significantly decreased in the intermediate-risk and high-risk patients $(\mathrm{p}<0.001$ and $\mathrm{p}<0.001$, respectively), whereas no significant differences were observed with the low-risk group $(\mathrm{p}=0.365)$. When AATD patients were compared to each other, telomerase activity was significantly lower in intermediate-risk and high-risk groups compared to the low-risk group $(\mathrm{p}<0.001, \mathrm{p}=0.001$; respectively). No significant differences in telomerase activity were observed between the high-risk and intermediate-risk groups $(\mathrm{p}=0.342)$.

\section{Oxidative stress markers are increased in AATD patients}

Biomarkers of oxidative stress in AATD patients and controls are shown in table 2. Significantly higher differences in biomarkers of oxidative stress, malonyldialdehyde (MDA; $\mathrm{p}<0.001$ ), 8 -hydroxydeoxyguanosine $(8-\mathrm{OHdG} ; \mathrm{p}<0.001)$ and protein carbonyls $(\mathrm{p}=0.01)$ were observed between groups. Intermediate-risk and high-risk patients showed significantly higher $\operatorname{MDA}(\mathrm{p}<0.01, \mathrm{p}<0.001)$, 8-OHdG $(p<0.01, p<0.001)$ and protein carbonyls $(p=0.01, p<0.01)$ than the control group. When compared to the control group, intermediate-risk and high-risk patients showed significantly decreased catalase activity $(\mathrm{p}<0.01, \mathrm{p}<0.001)$ and increased glutathione peroxidase activity $(\mathrm{p}<0.01, \mathrm{p}<0.001)$ leading to an accumulation of hydrogen peroxide $(\mathrm{p}<0.01, \mathrm{p}<0.001)$. No differences were observed between the control and the low-risk groups in any of the analysed parameters.

\section{Association between telomere length and oxidative stress markers and AAT levels in AATD} patients and in controls

A series of Spearman rank-order correlations were conducted in order to determine if there was a correlation between telomere length and oxidative stress markers in AATD patients and in controls. As telomere length is dependent on age, correlations were adjusted by age. As shown in table 3, in AATD patients there was a statistically significant negative correlation between telomere length and MDA (Rho $=-0.378, \mathrm{p}=0.043) ; 8-\mathrm{OHdG}$ ( $\mathrm{Rho}=-0.347, \mathrm{p}=0.041$ ) and $\mathrm{H}_{2} \mathrm{O}_{2}$ levels $(\mathrm{Rho}=-0.202, \mathrm{p}=0.008)$. No correlation between telomere length and protein carbonyls levels was observed $(\mathrm{Rho}=-0.061, \mathrm{p}=0.680$ ). There was a positive correlation between telomere

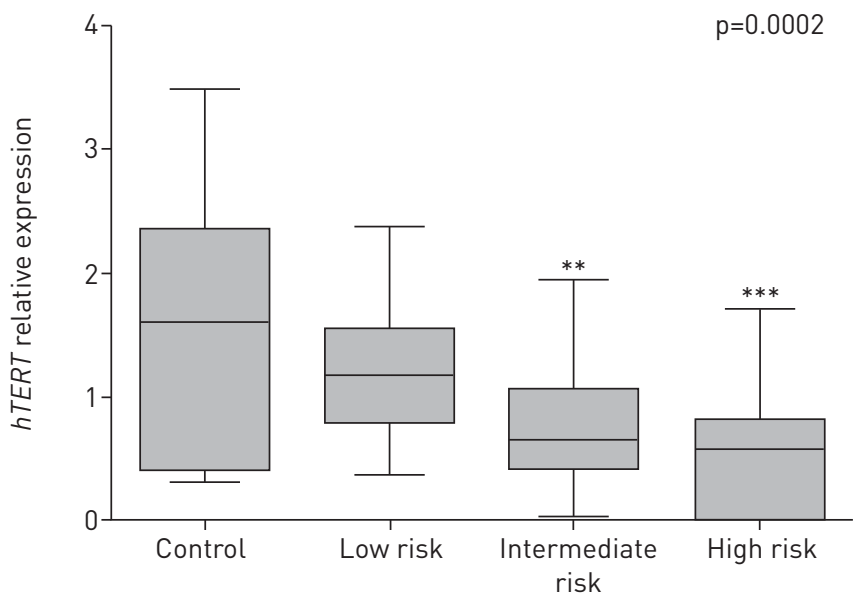

FIGURE 2 Decreased telomerase reverse transcriptase ( $h T E R T$ ) expression in peripheral blood mononuclear cells of $\alpha_{1}$-antitrypsin deficiency (AATD) patients. Boxplots show hTERT mRNA expression and AATD risk groups. Data are presented as median and interquartile range. Significant differences in $h T E R T$ expression were observed between groups ( $p=0.0002$ ). $h T E R T$ expression was significantly decreased in intermediate-risk $(p=0.001)$ and high-risk patients $(p=0.0005)$ compared to controls. No significant differences were observed between the low-risk patients and controls ( $p=0.225)$. ${ }^{* *}: p \leqslant 0.01 ;{ }^{* *}: p \leqslant 0.001$. 


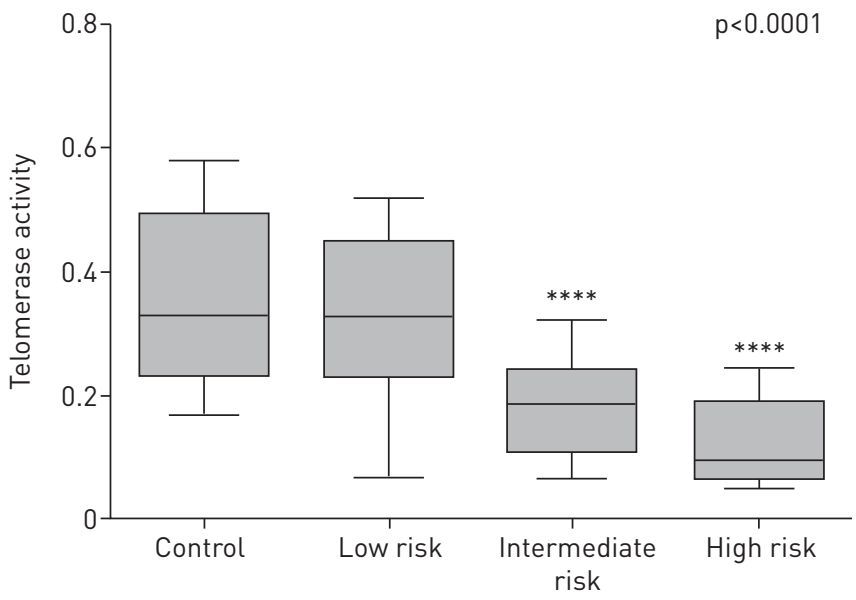

FIGURE 3 Decreased telomerase activity in peripheral blood mononuclear cells of $\alpha_{1}$-antitrypsin deficiency (AATD) patients. Boxplots show telomerase activity and AATD risk groups. Data are presented as median and interquartile range. Significant differences in telomerase activity were observed between groups $(p<0.0001)$. Telomerase activity was significantly decreased in intermediate-risk ( $p<0.001)$ and high-risk patients ( $p<0.001)$ compared to controls. No significant differences were observed between the low-risk patients and controls ( $p=0.365)$. ${ }^{* * * *}: p \leqslant 0.0001$.

length and AAT levels, which was statistically significant ( $\mathrm{Rho}=0.374 ; \mathrm{p}=0.002$ ). No correlation was observed between telomere length and oxidative stress parameters or AAT levels in controls (table 3).

\section{Discussion}

Recent studies by our research group have shown that oxidative stress is involved in the pathophysiology of AATD [7]. Throughout the years, much evidence has shown an association between oxidative stress and telomere shortening [23-25]. Telomeres (and telomerase) are involved in the pathogenesis of several lung diseases, including idiopathic pulmonary fibrosis, COPD, emphysema and lung cancer [12, 26]. One common characteristic in all these diseases is that patients have shorter telomeres than controls [27] and it has been reported that short telomere length is a genetic susceptibility factor for the development of emphysema [15-17]. Moreover, the association between lung function and telomere length has been addressed in several studies. Patients with moderate-to-severe COPD show significantly shorter telomeres than controls $[28,29]$. Short telomeres are a risk factor for idiopathic pulmonary fibrosis and are a determinant of cigarette smoking-induced emphysema susceptibility in mice $[16,26]$. Based on these evidences, we hypothesised that AATD patients would have shorter telomeres than healthy individuals and that telomere length would be related to AAT phenotypes and, therefore, to the risk of developing lung and/or liver damage in AATD patients.

\section{TABLE 2 Biomarkers of oxidative stress in $\alpha_{1}$-antitrypsin deficiency (AATD) and in control individuals}

\section{AATD patients}

p-value

Low risk (MS; SS) Intermediate risk (MZ; SZ)

\section{High risk (ZZ)}

\section{Subjects}

Oxidative stress markers

8-OHdG $\mathrm{ng} \cdot \mathrm{mL}^{-1}$

MDA pmol. $\mathrm{mg}^{-1}$

$\mathrm{PC}$ nmol. $\mathrm{mg}^{-1}$

$\mathrm{H}_{2} \mathrm{O}_{2}$ fluorescence

units

\section{Antioxidant capacity}

SOD activity

CAT activity

GPx activity

GR activity
18

$0.63(0.49-0.87)$
$0.91(0.56-1.35)$
$0.16(0.09-0.24)$
$229(74-425)$

5.30 (3.49-7.39)

$37.39(24.43-51.73)$

$21.36(13.96-29.56)$

$3.90(2.74-4.96)$
20

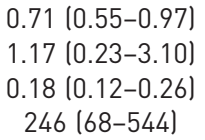

5.61 (3.83-8.95)

37.32 (21.42-57.93)

$22.62(18.21-29.14)$

$3.85(2.42-6.75)$
34

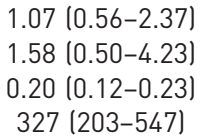

6.30 (3.82-8.95)

$27.52(10.73-44.21)$

24.75 (17.45-38.71)

$4.35(2.29-7.13)$
8

$\begin{array}{cc}1.77(0.80-2.58) & <\mathbf{0 . 0 0 1} \\ 2.59(0.92-6.17) & <\mathbf{0 . 0 0 1} \\ 0.23(0.19-0.27) & \mathbf{0 . 0 1} \\ 518(369-601) & <\mathbf{0 . 0 0 1}\end{array}$

$\begin{array}{cc}5.24(3.78-6.18) & 0.08 \\ 18.33(13.17-24.80) & <\mathbf{0 . 0 0 1} \\ 33.15(28.53-39.40) & <\mathbf{0 . 0 0 1} \\ 3.98(2.55-4.97) & 0.60\end{array}$

Data are presented as median (interquartile range), unless otherwise stated. Bold indicates statistical significance $(p<0.05)$ after application of the Kruskal-Wallis test. 8-OHdG: 8-hydroxydeoxyguanosine; MDA: malonyldialdehyde; PC: protein carbonyls; $\mathrm{H}_{2} \mathrm{O}_{2}$ : hydrogen peroxide; SOD: superoxide dismutase; CAT: catalase; GPx: glutathione peroxidase; GR: glutathione reductase. 
TABLE 3 Age-adjusted correlations between telomere length and oxidative stress markers and $\alpha_{1}$-antitrypsin (AAT) levels in $\alpha_{1}$-antitrypsin deficiency (AATD) patients and controls

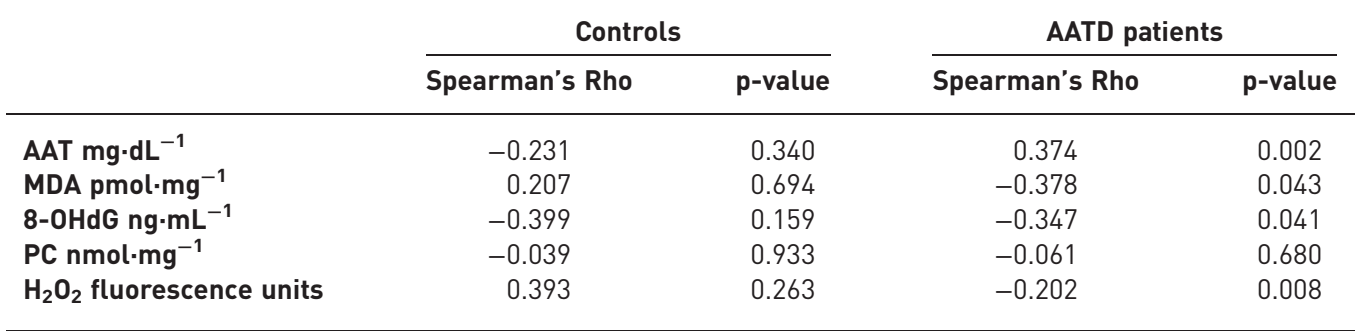

Correlations were assessed with Spearman's rank test. A p-value $<0.05$ was statistically significant. MDA: malonyldialdehyde; 8-OHdG: 8-hydroxydeoxyguanosine; PC: protein carbonyls; $\mathrm{H}_{2} \mathrm{O}_{2}$ : hydrogen peroxide.

The present study shows that telomeres of PBMC are significantly shorter in AATD patients than in healthy controls. The results show that intermediate- and high-risk patients have significantly shorter telomeres than healthy controls. This may be explained by the lower mRNA hTERT expression and decreased telomerase activity observed in these patients compared to those for the control group. Multiple hypothesis testing indicates an association between telomere length and AATD phenotypes. High-risk patients showed shorter telomeres, lower hTERT expression and decreased telomerase activity than intermediate-risk patients. This is similar to that observed between these patients and the low-risk patients. Nevertheless, no differences were observed either in telomere length, mRNA hTERT expression or telomerase activity between the low-risk and the control groups.

In order to support the hypothesis that oxidative stress leads to telomere attrition, assessment of oxidative stress markers was performed in AATD patients and control individuals. Our results indicate increased oxidative stress in AATD patients and are in agreement with our previous results [7]. Spearman's correlation analysis showed a negative correlation between telomere length and oxidative stress markers (MDA, 8-OHdG and $\mathrm{H}_{2} \mathrm{O}_{2}$ ), which is consistent with our hypothesis that increased oxidative stress causes accelerated telomere shortening in AATD patients. In addition, our results show for the first time a positive correlation between telomere length and AAT levels.

Telomere length has not been extensively studied in AATD. In the only study published to date, it was shown that COPD patients with AATD had longer telomeres in leukocytes than COPD patients without AATD. The authors also found that telomere length determined in blood may be an appropriate surrogate for telomere length in the lungs. However, there was no significant association between leukocyte telomere length and patient lung function [30]. Our study is not comparable to the study by SAFERALI et al. [30] since the design of both studies differed considerably. Our study was conducted in AATD healthy children and young adults versus adult AATD patients with COPD in the study by SAFERALI et al. [30]. Moreover, in that study there was no control group without COPD, therefore, it is not possible to establish whether patients with AATD present telomere shortening compared with healthy individuals. Also, the authors did not separate AATD patients by their AAT phenotypes. There is a direct association between the AAT levels and telomere length according to our study, thus its determination is important for the interpretation of the results. Finally, smoking may affect telomere length [14]. In the study by SAFERALI et al. [30], all AATD patients were current or former smokers, therefore, the authors were not able to test for the effect of AATD in nonsmokers compared with smokers.

Several possibilities could explain how oxidative stress shortens telomeres in AATD patients. First, decreased $h T E R T$ expression and telomerase activity would cause a decrease in catalase activity, leading to $\mathrm{H}_{2} \mathrm{O}_{2}$ accumulation and increasing $\mathrm{OS}$, which would downregulate telomerase activity leading to accelerated telomere shortening. A direct link between telomerase and catalase deficiency has been reported in animal models. Increased oxidative stress and oxidative damage, caused by higher $\mathrm{O}_{2}^{-}$and $\mathrm{H}_{2} \mathrm{O}_{2}$ production and lower catalase activity, have been previously reported in mouse embryonic fibroblasts and kidneys isolated from mice lacking telomerase activity $\left(\mathrm{Terc}^{-1-}\right)$. Restoration of telomerase activity in this model increases catalase expression and activity, lowering oxidative stress [13]. Secondly, it has been shown in human endothelial cells that chronic oxidative stress rapidly downregulates telomerase activity leading to telomere accelerated shortening [25].

In addition, since $\mathrm{H}_{2} \mathrm{O}_{2}$ is itself a powerful oxidant and central precursor to both $\mathrm{OH}$ and $\mathrm{HOCl}$ (two extremely potent oxidants), its accumulation would explain the significantly increased levels of oxidative stress biomarkers observed in high- and intermediate-risk patients. Due to its high content of guanines, telomeres 
are especially sensitive to the accumulation of single-strand breaks induced by 8-OHdG [31, 32], which will interfere with the replication fork leading to telomere shortening observed in these groups of patients.

It has long been known that $h T E R T$ gene expression correlates with telomerase activity, therefore the reduction in telomerase activity observed in AATD patients is probably caused by a decrease in $h T E R T$ expression and the resultant reduction of $h T E R T$ production. However, recent research has led to the characterisation of additional transcription factors and epigenetic mechanisms that play a role in hTERT expression [33]. For instance, it has been recently described that prolonged long-term exposure to endoplasmic reticulum stress (as happens in AATD) causes hTERT expression downregulation in tumour cells [34]. Therefore, the possibility that these factors could influence hTERT expression in AATD patients cannot be ruled out.

Whether telomerase activity reduction is a direct or indirect effect of low AAT levels is currently unknown. No evidences have been found of a direct regulation of telomerase activity by AAT, so current data point to an indirect regulation through regulation of oxidative stress and inflammation [35], hTERT expression [34] and iron metabolism [36].

Several factors, such as ageing, tobacco and alcohol consumption, and obesity (among many others) modify the oxidative stress status. The present study was conducted in children and young teenagers to avoid these confounding factors as much as possible. None of the participants included in the study were obese or had been exposed to cigarette smoke (according to familiar interviews). These aspects are of particular importance as they emphasise the fact that clinically healthy AATD individuals have alterations at the biochemical level that could be important in the prognosis of the disease.

As stated above, in humans, AATD shows a highly variable clinical phenotype and the existence of genetic modifiers that may influence disease progression has been proposed $[4,5]$. Our results are in agreement with these reports and suggest that it might be worth studying allelic variations of genes involved in glutathione metabolism, oxidative stress defence and telomere length regulation as potential genetic factors relevant to the severity of disease.

A major limitation of the present study is the limited number of homozygous ZZ patients analysed $(n=8)$, which is not surprising given the low prevalence of this phenotype. Although we are aware that this could be a problem for reaching definitive conclusions, the high significance of the differences found indicates that the results would probably not vary significantly after introducing a greater number of ZZ patients into the study.

Our results, together with the observation that lung alveolar integrity is compromised by telomere shortening in telomerase null mice [37], suggests that telomere length (produced by increased oxidative stress) in PBMC are promising biomarkers for AATD disease progression although further studies, including a higher number of patients at different progression stages, is needed to determine the clinical relevance of this observation.

\section{References}

de Serres F, Blanco I. Role of alpha-1 antitrypsin in human health and disease. J Intern Med 2014; 276: 311-335. Stoller JK, Aboussouan LS. A review of $\alpha 1$-antitrypsin deficiency. Am J Respir Crit Care Med 2012; 185: 246-259. Fregonese L, Stolk J. Hereditary alpha-1-antitrypsin deficiency and its clinical consequences. Orphanet J Rare Dis 2008; 3: 16

4 Marcus NY, Blomenkamp K, Ahmad M, et al. Oxidative stress contributes to liver damage in a murine model of alpha-1-antitrypsin deficiency. Exp Biol Med (Maywood) 2012; 237: 1163-1172.

5 Borzone GR, Liberona LF, Bustamante AP, et al. Differences in lung glutathione metabolism may account for rodent susceptibility in elastase-induced emphysema development. Am J Physiol Regul Integr Comp Physiol 2009; 296: R1113-R1123.

6 Papp E, Száraz P, Korcsmáros T, et al. Changes of endoplasmic reticulum chaperone complexes, redox state, and impaired protein disulfide reductase activity in misfolding $\alpha 1$-antitrypsin transgenic mice. FASEB $J$ 2006; 20: 1018-1020.

7 Escribano A, Amor M, Pastor S, et al. Decreased glutathione and low catalase activity contribute to oxidative stress in children with $\alpha-1$ antitrypsin deficiency. Thorax 2015; 70: 82-83.

8 Haycock PC, Heydon EE, Kaptoge S, et al. Leucocyte telomere length and risk of cardiovascular disease: systematic review and meta-analysis. BMJ 2014; 349: g4227-g4227.

9 Aubert G, Lansdorp PM. Telomeres and aging. Physiol Rev 2008; 88: 557-579.

10 Houben JM, Moonen HJ, van Schooten FJ, et al. Telomere length assessment: biomarker of chronic oxidative stress? Free Radic Biol Med 2008; 44: 235-246.

11 Correia-Melo C, Hewitt G, Passos JF. Telomeres, oxidative stress and inflammatory factors: partners in cellular senescence? Longev Healthspan 2014; 3: 1.

12 Holohan B, Wright WE, Shay JW. Cell biology of disease: telomeropathies: an emerging spectrum disorder. J Cell Biol 2014; 205: 289-299.

13 Pérez-Rivero G, Ruiz-Torres MP, Díez-Marqués ML, et al. Telomerase deficiency promotes oxidative stress by reducing catalase activity. Free Radic Biol Med 2008; 45: 1243-1251.

14 Savale L, Chaouat A, Bastuji-Garin S, et al. Shortened telomeres in circulating leukocytes of patients with chronic obstructive pulmonary disease. Am J Respir Crit Care Med 2009; 179: 566-571.

15 Houben JM, Mercken EM, Ketelslegers HB, et al. Telomere shortening in chronic obstructive pulmonary disease. Respir Med 2009; 103: 230-236. 
16 Alder JK, Guo N, Kembou F, et al. Telomere length is a determinant of emphysema susceptibility. Am J Respir Crit Care Med 2011; 184: 904-912.

17 Morlá M, Busquets X, Pons J, et al. Telomere shortening in smokers with and without COPD. Eur Respir J 2006; 27: 525-528.

18 American Thoracic Society, European Respiratory Society. American Thoracic Society/European Respiratory Society statement: standards for the diagnosis and management of individuals with alpha-1 antitrypsin deficiency. Am J Respir Crit Care Med 2003; 168: 818-900.

19 Zapletal A, Samanek M, Paul T. Upstream and total airway conductance in children and adolescents. Bull Eur Physiopathol Respir 1982; 18: 31-37.

20 Pellegrino R, Viegi G, Brusasco V, et al. Interpretative strategies for lung function tests. Eur Respir J 2005; 26 : 948-968.

21 Bals R, Koczulla R, Kotke V, et al. Identification of individuals with alpha-1-antitrypsin deficiency by a targeted screening program. Respir Med 2007; 101: 1708-1714.

22 Cawthon RM. Telomere measurement by quantitative PCR. Nucl Acids Res 2002; 30: e47-e47.

23 Saretzki G, Von Zglinicki T. Replicative aging, telomeres, and oxidative stress. Ann N Y Acad Sci 2002; 959: 24-29.

24 Von Zglinicki T. Oxidative stress shortens telomeres. Trends Biochem Sci 2002; 27: 339-344.

25 Kurz DJ, Decary S, Hong Y, et al. Chronic oxidative stress compromises telomere integrity and accelerates the onset of senescence in human endothelial cells. J Cell Sci 2004; 117: 2417-2426.

26 Armanios M, Blackburn EH. The telomere syndromes. Nat Rev Genet 2012; 13: 693-704.

27 Gansner JM, Rosas IO. Telomeres in lung disease. Transl Res 2013; 162: 343-352.

28 Lee J, Sandford AJ, Connett JE, et al. The relationship between telomere length and mortality in chronic obstructive pulmonary disease (COPD). PLoS One 2012; 7: e35567.

29 Albrecht E, Sillanpää E, Karrasch S, et al. Telomere length in circulating leukocytes is associated with lung function and disease. Eur Respir J 2014; 43: 983-992.

30 Saferali A, Lee J, Sin DD, et al. Longer telomere length in COPD patients with $\alpha 1$-antitrypsin deficiency independent of lung function. PLoS One 2014; 9: e95600.

31 Zglinicki von T, Pilger R, Sitte N. Accumulation of single-strand breaks is the major cause of telomere shortening in human fibroblasts. Free Radic Biol Med 2000; 28: 64-74.

32 Coluzzi E, Colamartino M, Cozzi R, et al. Oxidative stress induces persistent telomeric DNA damage responsible for nuclear morphology change in mammalian cells. PLoS One 2014; 9: e110963.

33 Daniel M, Peek GW, Tollefsbol TO. Regulation of the human catalytic subunit of telomerase (hTERT). Gene 2012; 498: 135-146.

34 Hosoi T, Inoue Y, Nakatsu K, et al. TERT attenuated ER stress-induced cell death. Biochem Biophys Res Commun 2014; 447: 378-382.

35 Stockley RA. The multiple facets of alpha-1-antitrypsin. Ann Transl Med 2015; 3: 130.

36 Kepinska M, Szyller J, Milnerowicz H. The influence of oxidative stress induced by iron on telomere length. Environ Toxicol Pharmacol 2015; 40: 931-935.

37 Lee J, Reddy R, Barsky L, et al. Lung alveolar integrity is compromised by telomere shortening in telomerase-null mice. Am J Physiol Lung Cell Mol Physiol 2009; 296: L57-L70. 